\title{
Preparation of ribosomes for SmFRET studies: a simplified approach
}

\author{
Bassem Shebl ${ }^{1}$, Drew E. Menke ${ }^{1}$, Min Pennella, Raghav R. Poudyal, Donald H. Burke, and Peter \\ V. Cornish* \\ Department of Biochemistry, University of Missouri, Columbia, MO, USA
}

\begin{abstract}
During the past decade, single-molecule studies of the ribosome have significantly advanced our understanding of protein synthesis. The broadest application of these methods has been towards the investigation of ribosome conformational dynamics using single-molecule Förster resonance energy transfer (smFRET). The recent advances in fluorescently labeled ribosomes and translation components have resulted in success of smFRET experiments. Various methods have been employed to target fluorescent dyes to specific locations within the ribosome. Primarily, these methods have involved additional steps including subunit dissociation and/or full reconstitution, which could result in ribosomes of reduced activity and translation efficiency. In addition, substantial time and effort are required to produce limited quantities of material. To enable rapid and large-scale production of highly active, fluorescently labeled ribosomes, we have developed a procedure that combines partial reconstitution with His-tag purification. This allows for a homogeneous single-step purification of mutant ribosomes and subsequent integration of labeled proteins. Ribosomes produced with this method are shown to be as active as ribosomes purified using classical methods. While we have focused on two labeling sites in this report, the method is generalizable and can in principle be extended to any non-essential ribosomal protein.
\end{abstract}

\section{Introduction}

A significant component of the central dogma involves the translation of the genetic code to proteins, which function in many structural and enzymatic roles. Translation is a ubiquitous process that is shared across all domains of life. The platform upon which proteins are made is the ribosome, which is assisted by various accessory factors in an elegant and complex mechanism. At $\sim 2.5 \mathrm{MDa}$, the bacterial ribosome is one of the largest macromolecular complexes of the cell. The ribosome, composed of two subunits (large $50 \mathrm{~S}$ and small $30 \mathrm{~S}$ ), is assembled from an interwoven mesh of ribosomal RNA (rRNA) and a total of 52 ribosomal proteins. Given the high degree of homology and absolute conservation of specific regions within the structure,

\footnotetext{
1 "The authors wish it to be known that, in their opinion, the first two authors should be regarded as joint First Authors".

*To whom correspondence should be addressed. Tel: 573-882-0443; Fax: Email: cornishp@missouri.edu
} 
the bacterial ribosome has served as a model for studying translation and more generally in understanding the structure and function of cellular macromolecular complexes [1]\}.

Since the elucidation of the first atomic resolution ribosome structures in the early 2000 s, translation has gained an increased interest in many fields including X-ray crystallography, cryoelectron microscopy, and single-molecule biophysics [1-4].These techniques among others have provided an expanded understanding of the different kinetics and conformational dynamics of translation machinery. In particular, single molecule Förster Resonance Energy Transfer (smFRET) experiments have allowed researchers to tap into uncharted territories in ribosome function and have uncovered key aspects of the mechanism of translation [5]. Due to the inherent nature of smFRET experiments and notably the direct detection of relative distance changes, bulk averaging can be overcome revealing real time visualization of complex pathways and conformational changes of the ribosome and translation factors. Thus, smFRET studies performed on the ribosome provide powerful insight, which complements structural studies [6-11]

In early pioneering smFRET experiments on the ribosome, transfer RNAs (tRNAs) were fluorescently labeled and were observed to spontaneously fluctuate between distinct conformational states $[7,12]$. These experiments confirmed previous results showing that tRNAs transition between a classical and hybrid state during translation and showed that $\mathrm{P}$ and $\mathrm{A}$ site tRNA can transition to the hybrid state independently $[7,12,13]$. While these experiments with labeled tRNAs have provided essential knowledge relative to the mechanism of translation, experiments with fluorescent dyes directly attached to the ribosome were necessary to gain insight into the conformational flexibility of the ribosome. Hybrid-labeling schemes emerged involving the labeling of a ribosomal protein with another dye on P-site tRNA [6] or on a translation factor $[14,15]$. In separate experiments, dual labeling of the ribosome was accomplished by either labeling ribosomal proteins or by hybridizing fluorescently labeled DNA to rRNA extensions within the ribosome $[16,17]$. These studies collectively provided researchers with the ability to directly observe conformational changes occurring during translation and how these changes are influenced by differences in tRNA amino acylation states and the presence of antibiotics and other factors $[8,9,18,19]$. Labeling the ribosome in this manner poses a substantial technical challenge due to the complexity of the structure and the necessity, in some cases, to perform partial or full reconstitution of the ribosome [20-22]. Additionally, the chosen labeling scheme needs to be sufficient to produce a homogenous sample with minimal disruption to the biological structure, limiting the perturbation to functional activity.

Driven by the need for highly active, fluorescently labeled ribosomes, different laboratories have adapted standard methodologies and developed a wide range of purification protocols. Choosing a specific purification protocol is application-dependent. The classical purification approach involves a series of ultracentrifugation steps to isolate ribosomes from 
cellular debris and other macromolecular complexes[23]. While this procedure is sufficient to generate pure ribosomal subunits, the difficulty has been to target a dye molecule to a specific protein in the large ribosome complex. Two separate approaches have been employed to approach this problem. First, researchers have been able to specifically knockout individual nonessential ribosomal proteins generating knockout strains. The protein is then purified separately, fluorescently labeled and reconstituted into the ribosome purified from the knockout strain [17]. However, in the case of essential proteins, genetic knockouts are lethal. The entire set of ribosomal proteins of the target subunit has to be purified individually, fluorescently labeled and fully reconstituted into a ribosomal subunit $[20,21]$. This has only found success in the context of the $30 \mathrm{~S}$ subunit and involves the purification of 20 small subunit ribosomal proteins and the $16 \mathrm{~S}$ rRNA. An alternate approach to labeling ribosomal proteins has been to label rRNA by first extending the rRNA to allow for this sequence to be hybridized to a short fluorescently labeled DNA oligonucleotide [16]. In both approaches, one or several ultracentrifugation steps are still necessary, which requires a significant time investment. Hence, there is a necessity for a more cost effective and timesaving approach that ultimately results in highly active ribosomes. For further discussion on the different labeling schemes used we refer the reader to the following reviews [5,24-26].

By exploiting the physical properties of ribosomes, techniques such as size exclusion chromatography [27] and hydrophobic interaction chromatography [28] have been used to purify ribosomes. Affinity-tag based chromatography [29-34] has gained much popularity among purification techniques considering the facility and specificity of the approach. Affinity-tag based methods for purifying ribosomes bearing mutations involve overexpression of a tagged ribosomal protein or rRNA and purifying the tagged ribosomes from a background of native / wild-type ribosomes [34-36]. A more recent approach for non-lethal mutants involves introducing a specific tag within the bacterial genome to the ribosomal L7/12 stalk producing a homogenous population of tagged ribosomes[31].

Here, we report a new hybrid method for producing fluorescently labeled ribosomes of high activity and larger yields than previous methods (Figure 1). We combine both the ease of His-tag purification with the ability to genetically knock out ribosomal proteins in the same strain. This provides the benefit of producing labeled ribosome samples in significantly less time and provides the opportunity to extend this methodology to labeling other non-essential ribosomal proteins. Further, we have extensively characterized fluorescently labeled ribosomes produced using this technique and show that they are as active as ribosomes purified using classical purification methods. 


\section{Materials and Methods}

\subsection{Preparation of linear DNA cassette for L7/L12 His-tag}

The procedure was adapted from Ederth et al.[31]. pET-28a was used as a template to amplify the kanamycin (kan) -resistant cassette by primers L12Kanfor1 and PolBKanrev1 (Table 1). The amplified DNA fragment from the first PCR was purified and used as the template for the second PCR using primers L12homofor and polBhomorev (Table 1). The final DNA fragment has 47 nucleotides homologous to the $3^{\prime}$ end of the E.coli rplL gene, minus the stop codon. A (CAC) 6 repeat coding for six Histidines was inserted before a stop codon TAA. The final DNA fragment has 45 nucleotides homologous to the DNA sequence after the $r p / L$ gene. The final PCR product was purified and used as linear DNA fragment for lambda Red recombineering (see below).

\subsection{Construction of the parent His-tagged strain}

Plasmid pKD46, a lambda Red recombinase expression plasmid under the araBAD promoter, was transformed into BW25113 [37]. A single colony was inoculated and electrocompetent cells were prepared by growing at $30^{\circ} \mathrm{C}$ with L-arabinose. The linear DNA fragment carrying the kanresistance gene with homologous ends to $r p / L$ was electroporated into the competent cells.

Colonies that successfully recombined with the linear DNA fragment were selected on kan plates at $37^{\circ} \mathrm{C}$. The positive insertion colonies were verified by PCR amplification and DNA sequencing of the insertion site. The resulting strain with the correct insertion of a C-terminal (His) 6 tag on ribosomal protein L7/L12 was named BD0012 (Table 2).

\subsection{Construction of the knockout strains}

\subsubsection{Single knockout mutants.}

The $\Delta$ S6 (JW4158) and $\Delta$ L9 (JW4161) knockout mutants were purchased from the Keio collection and used for the purification of $\Delta S 630 \mathrm{~S}$ and $\Delta \mathrm{L} 950 \mathrm{~S}$ subunits (Table 2 ) in the classical subunit purification protocol (see below).

\subsubsection{Generation of a His-tagged double ribosomal protein knockout.}

The $\Delta$ L9 knockout mutant (JW4161) transformed with pCP20 [37] was cured of its kan-resistance cassette. P1 Phage transduction (see below) was used to move the L7/L12 His-tagged mutation from BD0012 to the cured $\Delta \mathrm{L} 9$ mutant. The resultant strain ( $\Delta \mathrm{L} 9 \mathrm{~L} 7 / \mathrm{L} 12$ His-tagged mutant) was named BD0912 (Table 2). Direct generation of a $\Delta \mathrm{L} 9 \Delta \mathrm{S} 6 \mathrm{~L} 7 / \mathrm{L} 12$ His-tagged mutant strain was not possible due to the close proximity of S6 (rpsF) and L9 gene (rp/l) in the genome and the limited packaging capacity of the P1 phage. A new S6 deletion cassette was designed to replace rps $F$ with a chloramphenicol-resistance cassette in a similar fashion as performed for the L7/L12 His-tag (see above) using primers S6forCat1/S6revCat1 and S6for2/S6rev2 (Table 1). The transformation of the $\Delta S 6$ chloramphenicol-deletion cassette and expression of the pKD46 
system in the $\Delta \mathrm{L} 9 \mathrm{~L} 7 / \mathrm{L} 12$ His-tagged strain resulted in a new $\Delta S 6 \Delta \mathrm{L} 9, \mathrm{~L} 7 / \mathrm{L} 12$ His-tag strain (BD6012) (Table 2) with antibiotic resistance cassettes for kan and chloramphenicol (cam).

\subsection{3 $P 1$ phage transduction}

The P1 vir phage used was a gift from Bob Sauer (MIT). A detailed protocol can be accessed online (http://openwetware.org/wiki/Sauer:P1vir_phage_transduction). In brief, P1 vir phage was used in the transfer of genetic material from a donor strain to a recipient strain. This is accomplished by the unique mechanism by which $\mathrm{P} 1$ phage packages random fragments of the donor strain genome, $\sim 90,000 \mathrm{bp}$. The donor strain was transduced with P1 vir phage and left to shake at $37^{\circ} \mathrm{C}$ for $1-3$ hours. Chloroform was added to complete cell lysis producing a new phage lysate. The clarified phage lysate was reintroduced to the original donor strain. A minimum of two passages was performed to clear the phage of previously held genetic material. The resultant phage was used to infect the recipient strain for 1 hour at $37^{\circ} \mathrm{C}$ after which sodium citrate was added to prevent the reinfection of phage into $E$. coli. Colonies were screened for the presence of the donor's antibiotic-resistance marker.

\subsection{Purification of ribosomes}

\subsubsection{Classical purification of tight-coupled ribosomes and subunits}

Tightly-coupled $70 \mathrm{~S}$ ribosomes and/or purified $50 \mathrm{~S}$ and $30 \mathrm{~S}$ subunits were purified via preparative sucrose gradient density ultracentrifugation of clarified cell lysate from either wild-type (WT) or mutant strains as described previously $[17,20,38]$. For a detailed protocol and a list off buffers used (Table S1), refer to the supplementary information.

\subsubsection{Affinity purification of tight-coupled His-tagged ribosomes}

A fresh overnight culture of $E$. coli BW21153, bearing the desired mutation(s) (Table 2), was used to inoculate LB with kan $(50 \mu \mathrm{g} / \mathrm{ml})$. The culture was grown with shaking at $37^{\circ} \mathrm{C}$ until an $\mathrm{OD}_{600}$ of 0.6. The culture was left to cool on ice with occasional shaking. Cells were harvested by centrifugation in a Sorvall SLC-6000 at 3,000 RPM for $20 \mathrm{~min}$. The cell-pellet was briefly rinsed with cold lysis buffer $\mathrm{G}$ and stored at $-80^{\circ} \mathrm{C}$. The cell-pellet was resuspended in lysis buffer $\mathrm{G}$ and lysed using a French Press. The cell debris was spun down twice in a Sorvall SS-34 at 12,000 RPM for $20 \mathrm{~min}$ at $4^{\circ} \mathrm{C}$. The clarified lysate was filtered and loaded onto a GE Healthcare Hisprep FF 16/10 equilibrated with buffer $G$. The column was washed with 5 column volumes of buffer $G$ to baseline the $A_{260}$ reading and to ensure the complete clearance of the flow-through. Next, non-specific binders were washed off with wash buffer $\mathrm{H}$ until the $\mathrm{A}_{260}$ went back to the baseline. The sample was eluted with elution buffer $\mathrm{I}$. The elution fractions were pooled and dialyzed immediately $3 x$ into $1 \mathrm{~L}$ of buffer $\mathrm{G}$ for $30 \mathrm{~min}$ each at $4^{\circ} \mathrm{C}$ to remove the imidazole. The ribosomes were concentrated by ultracentrifugation at $150,000 \times \mathrm{g}$ for $2 \mathrm{~h}$ at $4^{\circ} \mathrm{C}$. The pelleted 
ribosomes were resuspended in buffer B for BD0012 ribosomes or buffer S for the BD6912 ribosomes. Ribosomes were flash frozen and stored at $-80^{\circ} \mathrm{C}$.

\subsubsection{Affinity Purification of 505 and 30 S Subunits}

Individual subunits can be purified using affinity purification via minor modifications to the previous section. The clarified lysate was loaded on the GE Healthcare Hisprep FF 16/10. After the $260 \mathrm{~nm}$ absorbance baselined, the $30 \mathrm{~S}$ was eluted by dissociation from the $50 \mathrm{~S}$ with buffer $\mathrm{J}$. The column was washed with wash buffer $\mathrm{H}$ and the His-tagged $50 \mathrm{~S}$ subunit was eluted using elution buffer I. Dialysis to remove imidazole was performed as described in the previous section. The purified subunits were concentrated by centrifugation at $80,000 \times \mathrm{g}$ for $21 \mathrm{~h}$ at $4^{\circ} \mathrm{C}$ and resuspended in the appropriate buffer or exchanged and concentrated with a Millipore Centricon ${ }^{\circledR}$.

\subsection{Cloning and purification of ribosomal proteins}

rpsF (S6 gene) and rpll (L9 gene) were cloned into pET-11a. Site-directed mutagenesis was performed to introduce a single cysteine at residues D41C for S6 and N11C for L9 [17]. The S6 D41C mutant was expressed in E. coli BL21 (DE3). Cultures were induced for 4 hours with $1 \mathrm{mM}$ IPTG. Cells were pelleted in an SLC-6000 Sorvall rotor at 3,000 $\mathrm{g}$ for 20 minutes and stored at $20^{\circ} \mathrm{C}$. Cells were resuspended in lysis buffer $\mathrm{K}$ and lysed by French Press. The lysate was then clarified at 12,000 RPM for 15 minutes in a Sorvall SS-34 rotor at $4^{\circ} \mathrm{C}$. The pellet containing inclusion bodies was then dissolved in buffer L. After the solubilization of the inclusion bodies, the sample was dialyzed three times against buffer $\mathrm{M}$ at $4^{\circ} \mathrm{C}$. The sample was then clarified by centrifugation at 12,000 RPM for 15 minutes in a Sorvall SS-34 rotor at $4^{\circ} \mathrm{C}$ and filtered through a $0.8 \mu \mathrm{m}$ cellulose acetate syringe filter. The protein was purified by a 100/10 Resource $Q$ anion exchange column with a $120 \mathrm{~mL}$ 0-22\% linear gradient of Buffer $\mathrm{L}$ in buffer M. S6 D41C was further purified by Hiload 26/60 Superdex prep grade in buffer $M$. The purified protein was then dialyzed into S6 labeling buffer $\mathrm{N}$, concentrated and flash frozen.

The L9 N11C mutant was expressed in E. coli BL21 (DE3) by growth in 1.5L LB to an

$\mathrm{OD}_{600}$ of $0.4-0.8$ at $37^{\circ} \mathrm{C}$. Cultures were induced for 4 hours with $1 \mathrm{mM}$ IPTG. Cells were pelleted in an SLC-6000 Sorvall rotor at $3,000 \times \mathrm{g}$ for 20 minutes and stored at $-20^{\circ} \mathrm{C}$. Cells were resuspended in lysis buffer $\mathrm{K}$ and lysed by French Press. The lysate was then clarified at 12,000 RPM for 15 minutes in a Sorvall SS-34 rotor at $4^{\circ} \mathrm{C}$. The pellet containing inclusion bodies was then dissolved in $10 \mathrm{~mL}$ of buffer $O$. After solubilizing the inclusion bodies, the sample was dialyzed $3 x$ against buffer $P$ at $4^{\circ} \mathrm{C}$. The sample was then clarified by centrifugation at 12,000 RPM for 15 minutes in an SS-34 rotor at $4^{\circ} \mathrm{C}$ and filtered through a $0.8 \mu \mathrm{m}$ cellulose acetate syringe filter. The protein was purified by a 100/10 Resource $S$ cation exchange column with a $120 \mathrm{~mL} 0-100 \%$ linear gradient of buffer O in buffer P. L9 N11C was further purified by Hiprep 
Butyl FF 16/10 using a 100 - $0 \%$ ammonium sulfate gradient in buffer Q. Protein obtained by this method was then dialyzed into L9 labeling buffer R, concentrated and frozen.

\subsection{Fluorescent labeling of ribosomal proteins}

50-200 $\mu \mathrm{M}$ of S6 D41C or L9 N11C was mixed with 2-fold excess of tris (2-carboxyethyl) phosphine (TCEP) and incubated at room temperature for 30 minutes $[17,20]$. Twenty-fold excess of sulfo-Сy3/Cy5 maleimide (Lumiprobe) was then added to the mixture and incubated for 2-4 hour at room temperature or $4^{\circ} \mathrm{C}$ overnight. The reaction was quenched by addition of $6 \mathrm{mM} \beta$ mercaptoethanol ( $\beta M E)$. A NAP-5 column (GE Healthcare Life Sciences) was used to separate the labeled protein from free dye. Fractions containing the labeled protein were pooled together and exchanged into either S6 reassociation buffer S or L9 reassociation buffer T. Purified, labeled protein was run on $16 \%$ SDS-PAGE. The attachment of a fluorescent dye to a small ribosomal protein results in a small mass shift in the gel. Therefore, labeling efficiency was determined by a combination of fluorescent imaging on a Biorad ChemiDoc and Coomassie-staining. Labeling efficiencies near $\sim 100 \%$ were obtained for both S6 and L9.

\subsection{Reconstitution and assembly of ribosomes}

\subsubsection{Assembly of S6/L9 classically purified ribosomes}

$1.2 \mathrm{nmoles}$ of $\Delta \mathrm{S} 630 \mathrm{~S}$ and $1.8 \mathrm{nmoles}$ of S6 D41C sulfo-Cy5 were assembled together in S6 reassociation buffer $\mathrm{S}$ at $37^{\circ} \mathrm{C}$ for 15 minutes. 1.2 nmoles of $\Delta$ L9 $50 \mathrm{~S}$ and 1.8 nmoles of L9 N11C sulfo-Cy3 were assembled together in $\mathrm{L} 9$ reassociation buffer $\mathrm{T}$ at $37^{\circ} \mathrm{C}$ for 15 minutes. Both subunits were then exchanged into $70 \mathrm{~S}$ reassociation buffer $\mathrm{U}$ using a 100K MWCO Millipore Centricon ${ }^{\circledR}$. The reconstituted $30 \mathrm{~S}$ and $50 \mathrm{~S}$ subunits were heat activated at $42^{\circ} \mathrm{C}$ for 10 minutes. The reconstituted subunits were mixed together and associated at $37^{\circ} \mathrm{C}$ for 20 minutes. The samples were layered on a $10-30 \%$ sucrose gradient in $70 \mathrm{~S}$ reassociation buffer $\mathrm{U}$. The gradients were centrifuged at $71,000 \times \mathrm{g}$ for $14 \mathrm{~h}$ in an SW41 rotor. The $70 \mathrm{~S}$ peak was conservatively collected and dialyzed into $70 \mathrm{~S}$ reassociation buffer $U$ by using a $100 \mathrm{~K}$ MWCO Millipore Centricon ${ }^{\circledR}$. The reassociation efficiency was determined by absorption measurements at 260,550 and 650 . Reassociation efficiencies near $\sim 100 \%$ were obtained. Purified ribosomes were diluted, aliquoted and frozen at $-80^{\circ} \mathrm{C}$.

\subsubsection{Assembly of S6/L9 His-tagged ribosomes}

1.2 nmoles of $\Delta S 6 / \Delta L 9 L 12$ His-tagged $70 S$ and 1.8 nmoles of L9 N11C sulfo-Cy3 were assembled together in $\mathrm{L} 9$ reassociation buffer $\mathrm{T}$ at $37^{\circ} \mathrm{C}$ for 15 minutes. The ribosomes were then exchanged into S6 reassociation buffer S. 1.8 nmoles of S6 D41C sulfo-Cy5 were added and the mixture was incubated at $37^{\circ} \mathrm{C}$ for 15 minutes. The reassociated ribosomes were then exchanged into $70 \mathrm{~S}$ reassociation buffer $\mathrm{U}$ by using a 100K MWCO Millipore Centricon ${ }^{\circledR}$. 
Reassociation efficiencies were assessed as mentioned above. Purified ribosomes were aliquoted and flash frozen at $-80^{\circ} \mathrm{C}$.

\subsection{Measurement of the growth rate}

All of the generated strains (Table 2) and WT parent strains were grown from overnight cultures. The cultures were grown with and without antibiotics ( $\mathrm{kan} 50 \mu \mathrm{g} / \mathrm{ml}$ and cam $35 \mu \mathrm{g} / \mathrm{m}$ ). The $\mathrm{OD}_{600}$ was monitored using a Biotek Synergy 2 Multi-Mode Reader over the course of 10 hours with Greiner Bio-One 96 well plates.

\subsection{Sucrose density-gradient analysis}

$300-1200$ pmoles of ribosomal $70 S, 50 S$ or 30 S subunits were layered onto a $10-30 \%$ sucrose gradient in buffer $\mathrm{W}$ in an SW41 rotor at $71,000 \times \mathrm{g}$ for $14 \mathrm{~h}$ at $4^{\circ} \mathrm{C}$. The gradients were pumped with $40 \%$ sucrose using a Brandel density gradient fractionator while monitoring the $A_{260}$ absorbance.

\subsection{Assessment of labeled protein incorporation}

Purified, reconstituted ribosomes were assayed on a 16\% SDS-PAGE. Fluorescent images were taken using a Biorad ChemiDoc to detect fluorescently labeled proteins (Cy3: Excitation 548/ Emission 563, and Cy5: Excitation 646/ Emission 662). The gel was Coomassie-stained for total protein.

\subsection{Preparation of translation factors, mRNA, and tRNAs}

tRNA ${ }^{\text {fMet }}$, tRNA $^{\text {Phe }}$ and tRNA ${ }^{\text {Tyr }}$ were purchased from MP Biomedicals, tRNA probes and Sigma, respectively. $\mathrm{fMet}_{\text {-tRNA }}{ }^{\mathrm{fMet}}$ and NAc-Phe-tRNA ${ }^{\text {Phe }}$ were aminoacylated using DEAE-purified S100 enzymes. Aminoacylated tRNAs were purified from the charging reaction and the extent of aminoacylation was verified by acid gel electrophoresis [39-41]. The messenger ribonucleic acid (mRNA) used for smFRET, m291, is a variant of T4 bacteriophage gene 32 (T4gp32) [42]. We used m301, a variant of $\mathrm{m} 291$, for filter binding, where the ATG codon was replaced with UAC $[17,42]$. The mRNA was in vitro transcribed from a PCR template with an upstream T7 promoter and purified on denaturing PAGE $[43,44]$. Elongation Factor-G (EF-G) was cloned from MRE600 into an expression vector with a C-terminus (His) $)_{6}$ tag. After overexpression in BL21, EF-G was purified on a GE Healthcare Hisprep FF 16/10 column and eluted in 250 mM imidazole. Purified EF-G was buffer exchanged to storage buffer $V$ and stored at $-80^{\circ} \mathrm{C}$ [45].

\subsection{2 tRNA-binding and puromycin reactivity assay}

The assay was performed as described previously with minor modifications $[5,46]$. To assay tRNA binding in the P-site, ribosomes were assembled with m301 and $\mathrm{N}-\mathrm{Ac}-\left[{ }^{3} \mathrm{H}\right]$ Phe - 
tRNA ${ }^{\text {Phe }}$ and incubated at $37^{\circ} \mathrm{C}$ for $20 \mathrm{~min}$. All samples were bound to a nitrocellulose membrane (Millipore HAWP $0.45 \mu \mathrm{m}$ ) and washed $3 \mathrm{x}$ with buffer $\mathrm{X}$ and the membranes were left to dry. All membranes were counted in scintillation cocktail (Bio-Safe $I^{\mathrm{TM}}$, RPI). For assessing tRNA binding in the A-site, ribosomes were incubated with tRNA ${ }^{\mathrm{Tyr}}$ at $37^{\circ} \mathrm{C}$ for $20 \mathrm{~min}$, followed by a second incubation with $\mathrm{N}-\mathrm{Ac}-\left[{ }^{3} \mathrm{H}\right]$ Phe $-\mathrm{tRNA} \mathrm{A}^{\mathrm{Phe}}$ at $37^{\circ} \mathrm{C}$ for $20 \mathrm{~min}$. To assay peptidyl-transferase center (PTC) activity, ribosomes were assembled with $\mathrm{N}-\mathrm{Ac}-\left[{ }^{3} \mathrm{H}\right] \mathrm{Phe}-\mathrm{tRNA}{ }^{\text {Phe }}$ as mentioned above, and incubated with puromycin, an antibiotic, to a final concentration of $1 \mathrm{mM}$ at $37^{\circ} \mathrm{C}$ for $10 \mathrm{~min}$. The reaction was quenched with an equivalent volume of $0.3 \mathrm{M} \mathrm{NaOAc}(\mathrm{pH} 5.4)$, saturated with $\mathrm{MgSO}_{4}$. Puromycin-bound $\left[{ }^{3} \mathrm{H}\right]$ Phe was extracted using $1 \mathrm{ml}$ of ethyl acetate and $800 \mu \mathrm{l}$ of the organic phase was counted. Finally, EF-G translocation efficiency was assayed by preassembling the ribosome with $t R N A^{T y r}$ and $N-A c-\left[{ }^{3} \mathrm{H}\right]$ Phe $-t R N A^{\text {Phe }}$ and incubating with EF-G/GTP at $37^{\circ} \mathrm{C}$ for $15 \mathrm{~min}$. Subsequently, puromycin was added as mentioned above to assess the P-site bound $\mathrm{N}-\mathrm{Ac}-\left[{ }^{3} \mathrm{H}\right]$ Phe $-\mathrm{tRNA}^{\text {Phe }}$ fraction.

\subsection{Preparation of ribosomal complexes for smFRET experiments.}

All ribosome complexes used for smFRET imaging were assembled in buffer $Y$ ( 20 mM HEPES$\mathrm{KOH} \mathrm{pH} \mathrm{7.5,} 6 \mathrm{mM} \mathrm{MgCl}_{2}, 150 \mathrm{mM} \mathrm{NH}_{4} \mathrm{Cl}, 6 \mathrm{mM} \mathrm{\beta ME}, 2 \mathrm{mM}$ spermidine, and $0.1 \mathrm{mM}$ spermine) [8]. The presence of polyamines, spermidine and spermine, stabilizes ribosomal subunit association even at low Mg2+ concentrations [47,48]. Ribosomal complexes carrying a single tRNA bound to the P-site were assembled by incubating $1 \mu \mathrm{M}$ S6/L9 labeled ribosomes with $2 \mu \mathrm{M}$ m291, pre-annealed to biotin-labeled DNA primer (5' biotin, CTTTATCTTCAGAAGAAAAACC-3', Integrated DNA Technologies), and $2 \mu \mathrm{M}$ tRNA (tRNA ${ }^{\mathrm{fMet}}$ or fMet-tRNA ${ }^{\mathrm{fMet}}$ ) at $37^{\circ} \mathrm{C}$ for $20 \mathrm{~min}$. The pre-translocation complexes were assembled by incubation of $1 \mu \mathrm{M}$ S6/L9 labeled ribosomes with $2 \mu \mathrm{M}$ m291 pre-annealed to biotin-labeled DNA primer, and $2 \mu \mathrm{M} \mathrm{tRNA}{ }^{\mathrm{fMet}}$, at $37^{\circ} \mathrm{C}$ for 20 min followed by a second incubation with $2 \mu \mathrm{M} \mathrm{N}$-Ac-Phe-tRNA ${ }^{\text {Phe }}$ at $37^{\circ} \mathrm{C}$ for 20 min.

Quartz slides were cleaned with acetone, methanol and $1 \mathrm{M} \mathrm{KOH}$ in sequence [25,49-51]. The surface was passivated with a mixture of $\mathrm{m}$-PEG - succinimidyl valerate (MW 5000) and Biotin - PEG - succinimidyl valerate (MW 5000) (Laysan Bio Inc.) and pretreated with neutravidin $(0.2 \mathrm{mg} / \mathrm{mL})$ (Pierce). Ribosomal complexes were diluted to a final concentration of $1 \mathrm{nM}$ and immobilized on a quartz slide. To minimize photobleaching, all samples were imaged in buffer $Y$ supplemented with an oxygen-scavenging system; $0.8 \mathrm{mg} / \mathrm{mL}$ glucose oxidase (Sigma), 0.625\% $\beta$-D-glucose, $0.02 \mathrm{mg} / \mathrm{mL}$ catalase (Roche), and $1.5 \mathrm{mM}$ Trolox (Sigma).

\subsection{4 smFRET data acquisition and analysis}

smFRET measurements were acquired on an Olympus IX-71 microscope with a UPlanSApo 60XW 1.20 objective lens. A $532 \mathrm{~nm}$ laser (Spectra-Physics) was used for excitation of Cy3 via prism-type total internal reflection microscopy (TIRFM) [25,50]. The fluorescence emission was 
split in two pathways (Cy3, Cy5 emission) with a 630dcxr dichroic mirror (Chroma). For visualizing the fluorescence signal, an Andor iXon ${ }^{\mathrm{EM}}+897$ EMCCD camera was used. Movies were recorded using Single (software downloaded from Taekjip Ha's lab website: https://cplc.illinois.edu/software/). The data was acquired at 100 milliseconds for all constructs. A calibration image was acquired using crimson fluorescent FluoSpheres ${ }^{\circledR}$ (Invitrogen). This allows for mapping of the two channels and accounts for rescaling, rotation and shear distortion. The recorded movies were processed to extract data using custom IDL scripts (Exelisvis), which were subsequently analyzed using custom scripts in Matlab (Mathworks).

Selection criteria for valid traces included: choosing traces showing single step photobleaching for Cy3 and Cy5, the trace lasts for $>1$ second and either showed a stable FRET or anti-correlated behavior. Traces showing positive correlation, fluctuating dye effect, an intensity less than 100 arbitrary units (au) or FRET states less than 0.15 were excluded. FRET histograms were constructed from traces fitting the set criteria. The contribution of each trace to the histogram was normalized to the length of the trace to ensure equal contribution to the final histogram $[49,52]$. Data were smoothed with a five-point window average. The histograms were fit to two Gaussian distributions using IGOR Pro (Wavemetrics).

\section{Results and Discussion}

Through the experimental procedures as described in Materials and Methods, we constructed a mutant bacterial strain that contained a genetically encoded His-tag on the L7/L12 stalk and also contained genomic knockouts of both rpsF and $r p l l$, which encode for ribosomal proteins $\mathrm{S} 6$ and L9, respectively. From this strain, ribosomes were purified through His-tag chromatography and partially reconstituted with fluorescently labeled S6 and L9 to generate S6 (Cy5) / L9 (Cy3) Histagged ribosomes for use in fluorescence based studies. Due to the high conservation within the ribosome, concern for potential perturbations that may result from mutagenesis, and the partial reconstitution, a thorough assessment of the generated mutant strains and fluorescently labeled ribosomes was necessary. We assessed the impact of introducing a genomic tag on the vitality of the mutated strains and the quality of the purified ribosomes in terms of intactness, purity, and most importantly the biochemical activity related to protein synthesis. Finally, we assessed the feasibility of using the new construct in performing smFRET experiments. To compare these results with previous methods of preparing fluorescently labeled ribosomes, we purified $30 \mathrm{~S}$ and 50 S subunits separately using ultracentrifugation, partially reconstituted with fluorescently labeled S6 and L9 ribosomal proteins, and reassembled 30 S and 50 S subunits to construct 70 S ribosome particles. 


\subsection{Construct design and rationale}

L7/L12 is unique in that the protein is present on the ribosome in four copies. L7/L12 constitutes a crucial part of the ribosomal L7/L12 stalk, believed to be involved in recruiting translation factors and subsequent GTPase activity. The N-terminal domain (NTD) of L7/L12 is involved in proteinprotein interaction with L10, another ribosomal protein [53,54]. L10 anchors the tetramer and interfaces with rRNA on the 50S subunit. Structural analysis shows the C-terminal domain (CTD) is linked to the NTD via a flexible hinge region. The flexibility of this region is required for conformational changes during translation. Structural analysis of L12 shows a strictly conserved region on the CTD involved in binding to different translation factors. However, the extreme terminus of the CTD region was not found to be involved in any binding interactions and does not require the rotational freedom of the NTD. Thus, it is a good candidate for the insertion of an affinity tag for rapid purification. Indeed, researchers were able to incorporate a genomic His-tag in the $L 7 / L 12$ gene ensuring that the produced ribosomes are homogeneously tetra - $(\mathrm{His})_{6}-$ tagged. Further, they showed, that the tetra-tag was highly specific and provided strong binding of ribosomes in cell lysate to a nickel affinity column [31].

To expedite both purification and fluorescent labeling of ribosomes, we decided to make genomic knockouts in the same strain containing a His-tag on the L7/L12 stalk. While any nonessential ribosomal protein can in principle be directed for labeling, we decided to target proteins S6 and L9 (Figure 2, PDB ID: 5AFI) [55]. Ribosomes containing fluorescent dyes on proteins S6 and L9 have been well characterized by our studies and others to investigate intersubunit rotation and conformational dynamics $[8,17,49,56]$. The ribosome samples in these previous studies have been prepared by classical methods using partial and full reconstitution. However, in all cases 30 S and 50 S subunits were prepared separately and reassembled, which is known to result in reduced activity $[17,20]$.

\subsection{Growth rate determination}

To measure the changes in growth rate as a result of the introduced genomic mutations, we grew all strains including controls in a temperature regulated plate reader as described in materials and methods. MRE600, which lacks ribonuclease I, is a commonly used $E$. coli strain for ribosome preparation, and BW25113 is the parent strain for the Keio collection. When compared to the parent strain BW25113, the L12 His-tagged strain (BD0012) showed a comparable growth rate consistent with previous results with His-tagged ribosomes (Figure 3A) [31]. For single knockout mutants, $\Delta S 6$ or $\Delta \mathrm{L} 9$, (JW4158 or JW4161, respectively) the growth rate was not significantly affected. However, the double knockout mutants (BD6900 or BD6912) required nearly double the time to reach the same OD as the parent strain. While non-essential for cell survival, the S6/L9 deletion in both strains could potentially have a negative effect on translation through reduced 
translation efficiency or ribosome assembly. Specific mutations or the complete absence of L9 is thought to interfere with the precise arrangement of the P-site tRNA [57]. S6 has no known function independent of binding directly to 16S rRNA in the 30S subunit near the E-site [58]. However, it is clear that the cumulative effect of both deletions is not lethal. Since the stability and integrity of the ribosome is restored after reconstitution with S6 and L9 as shown below, the reason for reduced growth efficiency was not pursued further. The growth rate of all strains is not affected with the addition of antibiotics, kanamycin and chloramphenicol, to the cultures, which shows the stability of the deletion strains as evidenced by the maintenance of the antibioticresistance cassettes. (Figure 3B). Finally, tagging the L7/L12 stalk with a tetra - $(\mathrm{His})_{6}-$ tag did not affect the apparent growth rate of the WT strain. Also, there was no difference between the growth rate for BW6912 and BD6900, which only differ by a His-tag on the L7/L12 stalk.

\subsection{Sucrose gradient analysis}

Similar to other reports, classically purified tight-coupled ribosomes under our purification conditions show a significant 50 S peak along with a 70S peak (Figure S1). The presence of $50 \mathrm{~S}$ subunits indicates the dissociation of loosely bound subunits breaking up the 70S ribosome. In a classical subunit preparation, the magnesium concentration is lowered to dissociate the two subunits followed by a sucrose gradient purification to individually purify the two subunits $[34,38,59]$. Sucrose gradient peaks have to be collected conservatively to avoid crosscontamination, which has the negative consequence of obtaining a lower yield. As an example when purifying $70 \mathrm{~S}$ ribosomes as in Figure S1, we selected only those fractions that would minimize $50 \mathrm{~S}$ contamination. This resulted in a loss of $\sim 10 \%$ of the total $70 \mathrm{~S}$ ribosomes present in the gradient. Additionally, $\sim 23 \%$ of 70 s ribosomes are lost due to dissociation into component subunits.

To assess the purity of His-tagged WT ribosomes and subunits, we performed a sedimentation analysis using ultracentrifugation. These ribosomes or dissociated subunits were loaded on a $10-30 \%$ analytical scale sucrose gradient. In contrast to tight-coupled classically purified ribosomes, His-tagged preparations, whether tight-coupled or subunit preparations, show primarily a single peak on the gradients (Figure 4). Hence, His-tagged ribosomes show tighter subunit coupling compared to a classical preparation. Potentially, the constant exposure to elevated magnesium levels throughout the affinity-purification, reduced purification time, and the lack of ultracentrifugation preserves the integrity of the ribosomes in this context. Also, when performing a subunit preparation using His-tag purification, we were able to observe nearly complete dissociation of $50 \mathrm{~S}$ and $30 \mathrm{~S}$ subunits providing homogeneous samples of each subunit. Thus, affinity-purification preserves the integrity of ribosomes during purification and provides a superior method for separating dissociated subunits. 


\subsection{D-gel analysis}

To compare the purity and consistency of the purified and/or reconstituted ribosomes, samples were denatured and subjected to SDS-PAGE (Figure 5) [59]. S1 is the largest ribosomal protein and the only protein migrating above the $35 \mathrm{kDa}$ marker (Figure 5A) [60]. The Coomassiestained gel shows a comparable pattern among the four constructs, A through $D$, with proteins grouping mostly in two major sets as shown previously [59]. When the gel was scanned for the presence of Cy3 and Cy5, fluorescent bands were present in the two center lanes, B and C, consistent with the molecular weights of Cy5-S6 and Cy3-L9 for both classically purified and Histagged ribosomes (Figure 5B). The protein-banding pattern is consistent across the four constructs and shows high purity with no crude cellular proteins. Further, this clearly shows that the fluorescently labeled proteins S6 and L9 were reconstituted into the purified knockout mutant ribosomes.

\section{5 tRNA binding, PTC activity and EF-G translocation efficiency}

To assess tRNA binding efficiency and the integrity of both $P$ and $A$ sites, we employed radiolabeled $\mathrm{N}-\mathrm{Ac}-\left[{ }^{3} \mathrm{H}\right]$ Phe $-\mathrm{tRNA}{ }^{\text {Phe }}$ using filter binding as described in Materials and Methods. The results are expressed as a percentage of the efficiency/activity of the classically purified WT ribosomes (Figure 6, Table S2). tRNA binding efficiency of His-tagged ribosomes is comparable for both $\mathrm{P}$ and A sites with respect to classically purified WT ribosomes [31]. Previously, S6/L9 ribosomes were constructed by full reconstitution of the $30 \mathrm{~S}$ and partial reconstitution of the $50 \mathrm{~S}$ with subsequent reassembly of the subunits to form $70 \mathrm{~S}$ ribosomes. It was reported that tRNA binding efficiency was $60 \%$ of WT ribosomes. In this report, we perform partial reconstitution for both S6 and L9 with either classically purified 30S and 50S subunits similar to more recent reports [22] or with intact 70S His-tagged ribosomes. We observed minimal differences within error in all cases between the fluorescently labeled ribosomes and WT classically or His-tag purified ribosomes. This suggests that the His-tagged fluorescently labeled ribosomes described here are identical within error to WT ribosomes in terms of tRNA binding efficiency.

Next, ribosomes were tested for PTC activity by the addition of puromycin, which is an antibiotic that resembles a portion of the 3 ' end of an aminoacyl tRNA [13]. Puromycin reacts preferentially with $P$ site aminoacyl tRNA over A site aminoacyl tRNA. Radiolabeled $\mathrm{N}-\mathrm{Ac}-\left[{ }^{3} \mathrm{H}\right]$ Phe $-t R N A^{\text {Phe }}$ was added to the P-site for each ribosomal constructs with subsequent addition of puromycin as described in Materials and Methods. The PTC activity was normalized against the P-site tRNA binding efficiency of the corresponding construct. When comparing PTC activity, we observe comparable activity between WT classically purified and His-tag purified ribosomes. In 
contrast to this, there was a drop to $\sim 70 \%$ activity for the S6/L9 labeled constructs. The reason for this is unclear, but S6 and/or L9 may have some unknown role in PTC activity.

Finally, translocation efficiency was verified by providing tRNA ${ }^{\text {Tyr }}$ to the P-site and radiolabeled $\mathrm{N}-\mathrm{Ac}-\left[{ }^{3} \mathrm{H}\right]$ Phe $-\mathrm{tRNA}{ }^{\text {Phe }}$ to the A-site. EF-G.GTP was added as described and translocation efficiency was assessed by the addition of puromycin. In this case, all three constructs, WT His-tagged ribosomes, S6/L9 classically purified ribosomes, and S6/L9 His-tag purified ribosomes, exhibited reduced translocation efficiency as compared to WT classically purified ribosomes. However, the translocation efficiency of S6/L9 His-tag purified ribosomes showed the highest activity with translocation efficiency of $\sim 90 \%$. The apparent drop in translocation efficiency observed for the WT His-tagged ribosomes could result from batch-tobatch variability (see Supplementary Figure S2). However, the data does not suggest that EF-G translocation efficiency drops due to the presence of a His-tag on the L7/L12 stalk as evidenced with the normal translocation efficiency shown for the His-tagged S6/L9 mutant and as shown previously[17]. Further, translocation is improved over previously reported reconstituted ribosomes [17].

\section{6 smFRET measurements}

To test fluorescently labeled S6 (Cy5)/L9 (Cy3) ribosome activity in smFRET experiments, Histagged and classically purified ribosomes were prepared in defined translation states as previously investigated [8]. The fluorescently labeled ribosomes were assembled at equilibrium conditions as described with a deacylated tRNA in the P-site, an amino acylated tRNA in the Psite or a deacylated tRNA in the P-site and an amino acylated tRNA in the A-site. The data were analyzed and traces exhibiting FRET were selected and combined into FRET histograms (Figure 7). As observed previously, the histograms were best fit to two FRET states corresponding to a $\sim 0.4$ FRET rotated state and a $\sim 0.55$ FRET non-rotated state (Figure 8). Based on the fitted histograms, we calculated the percentage of molecules residing in either state, rotated versus non-rotated. The acylation status of the P-site tRNA dictates the status of the majority of molecules [8]. Next, we calculated the equilibrium constant as the ratio between the rotated and non-rotated molecules. These data indicate that classically and His-tag purified ribosomes are identical and the results are consistent with previous observations (Table 2). Additionally, traces that transitioned between these two states were observed (Figure 8). As before, the number of traces that fluctuate in a particular state is indicative of the dynamicity of the system. Again, the results are comparable between classical and His-tag purified ribosomes and previous reports. In general, the results are consistent with minor discrepancies between published and acquired data likely due to individual variations in data acquisition and sample preparation. 


\section{Conclusions}

Here, we present a faster, cheaper and simpler method of purifying and labeling ribosomes for fluorescence-based studies. The method presented makes it accessible to a wider audience of researchers as many researchers may lack the equipment for purifying ribosomes using classical methodology. In our experience, the preparation time is significantly reduced to as little as one day (as opposed to four days) with a sample yield of 2 to 3 times more than with the classical purification methods (Figure 1). Also, when purifying ribosomes using the His-tag method, contamination of other subunits is significantly reduced or eliminated as compared to classical methods. Due to minimal sample manipulation and a gentler purification approach, the purified ribosomes are as good as, if not more active than, ribosomes purified by ultracentrifugation. Our studies and others have shown that antibiotics bound to the ribosome can significantly alter the conformational dynamics of the ribosome $[8,61,62]$. Thus, we envision that fluorescence based high-throughput screens could be a viable option for screening for new target molecules. The methodology developed here would provide the sample quantity and quality necessary for such experiments. In addition, using the His-tagged construct in conjunction with the labeling of ribosomal proteins $\mathrm{S} 6$ and $\mathrm{L} 9$, homogenous samples of different functional translation complexes produced from crude translation lysates could be easily pulled down and investigated with SmFRET. It should be restated that in principle any non-essential ribosomal protein could be targeted for labeling for a fluorescent dye or any other molecule of interest. This would open new possibilities for purifying ribosome samples for contrast matching experiments in Small Angle Neutron Scattering (SANS), electron paramagnetic resonance (EPR) experiments, or even pursuing NMR based studies of individual ribosome subunits. Finally, the same principles could be applied to other cellular macromolecular complexes; facilitating the purification and labeling procedure.

\section{Acknowledgments}

The authors thank Bob Sauer for providing the P1 Phage. This work was supported by National Science Foundation CAREER award [grant number: MCB-115343] to P.V.C. P.V.C is a Pew Scholar in the Biomedical Sciences. Funding for open access charge: National Science Foundation. 


\section{References}

[1] A. Korostelev, D.N. Ermolenko, H.F. Noller, Structural dynamics of the ribosome, Current Opinion in Chemical Biology. 12 (2008) 674-683. doi:10.1016/j.cbpa.2008.08.037.

[2] J. Frank, R.K. Agrawal, A ratchet-like inter-subunit reorganization of the ribosome during translocation, Nature. 406 (2000) 318-322. doi:10.1038/35018597.

[3] M.M. Yusupov, Crystal Structure of the Ribosome at 5.5 A Resolution, Science. 292 (2001) 883-896. doi:10.1126/science.1060089.

[4] B.T. Wimberly, D.E. Brodersen, W.M. Clemons, R.J. Morgan-Warren, A.P. Carter, C. Vonrhein, et al., Structure of the 30S ribosomal subunit, Nature. 407 (2000) 327-339. doi:10.1038/35030006.

[5] B. Shebl, Z. Norman, P.V. Cornish, Ribosome Structure and Dynamics by smFRET Microscopy, 1st ed., Elsevier Inc, 2014. doi:10.1016/B978-0-12-801122-5.00016-7.

[6] J. Fei, P. Kosuri, D.D. MacDougall, R.L. Gonzalez Jr, Coupling of Ribosomal L1 Stalk and tRNA Dynamics during Translation Elongation, Molecular Cell. 30 (2008) 348-359. doi:10.1016/j.molcel.2008.03.012.

[7] S.C. Blanchard, H.D. Kim, R.L. Gonzalez, J.D. Puglisi, S. Chu, tRNA dynamics on the ribosome during translation, Proc. Natl. Acad. Sci. U.S.a. 101 (2004) 12893-12898. doi:10.1073/pnas.0403884101.

[8] P.V. Cornish, D.N. Ermolenko, H.F. Noller, T. Ha, Spontaneous Intersubunit Rotation in Single Ribosomes, Molecular Cell. 30 (2008) 578-588. doi:10.1016/j.molcel.2008.05.004.

[9] P.V. Cornish, D.N. Ermolenko, D.W. Staple, L. Hoang, R.P. Hickerson, H.F. Noller, et al., Following movement of the L1 stalk between three functional states in single ribosomes, Proceedings of the National Academy of Sciences. 106 (2009) 2571-2576. doi:10.1073/pnas.0813180106.

[10] X. Chen, H. Kang, L. Shen, M. Chamorro, A characteristic bent conformation of RNA pseudoknots promotes-1 frameshifting during translation of retroviral RNA, Journal of Molecular .... (1996).

[11] C. Chen, H. Zhang, S.L. Broitman, M. Reiche, I. Farrell, B.S. Cooperman, et al., Dynamics of translation by single ribosomes through mRNA secondary structures, Nat Struct Mol Biol. 20 (2013) 582-588. doi:10.1038/nsmb.2544.

[12] J.B. Munro, R.B. Altman, N. O'Connor, S.C. Blanchard, Identification of Two Distinct Hybrid State Intermediates on the Ribosome, Molecular Cell. 25 (2007) 505-517. doi:10.1016/j.molcel.2007.01.022.

[13] D. Moazed, H.F. Noller, Intermediate states in the movement of transfer RNA in the ribosome, Nature. 342 (1989) 142-148. doi:10.1038/342142a0.

[14] J.B. Munro, R.B. Altman, C.-S. Tung, K.Y. Sanbonmatsu, S.C. Blanchard, A fast dynamic mode of the EF-G-bound ribosome, Embo J. 29 (2010) 770-781. doi:10.1038/emboj.2009.384.

[15] E. Salsi, E. Farah, J. Dann, D.N. Ermolenko, Following movement of domain IV of elongation factor $\mathrm{G}$ during ribosomal translocation, Proceedings of the National Academy of Sciences. (2014). doi:10.1073/pnas.1410873111.

[16] M. Dorywalska, S.C. Blanchard, R.L. Gonzalez, H.D. Kim, S. Chu, J.D. Puglisi, Sitespecific labeling of the ribosome for single-molecule spectroscopy, Nucleic Acids Research. 33 (2005) 182-189. doi:10.1093/nar/gki151.

[17] D.N. Ermolenko, Z.K. Majumdar, R.P. Hickerson, P.C. Spiegel, R.M. Clegg, H.F. Noller, Observation of Intersubunit Movement of the Ribosome in Solution Using FRET, Journal of Molecular Biology. 370 (2007) 530-540. doi:10.1016/j.jmb.2007.04.042.

[18] D.N. Ermolenko, P.V. Cornish, T. Ha, H.F. Noller, Antibiotics that bind to the A site of the large ribosomal subunit can induce mRNA translocation, Rna. 19 (2013) 158-166. doi:10.1261/rna.035964.112.

[19] R.A. Marshall, M. Dorywalska, J.D. Puglisi, Irreversible chemical steps control intersubunit dynamics during translation, Proceedings of the National Academy of 
Sciences. 105 (2008) 15364-15369. doi:10.1073/pnas.0805299105.
R. Hickerson, Z.K. Majumdar, A. Baucom, R.M. Clegg, H.F. Noller, Measurement of Internal Movements within the 30S Ribosomal Subunit Using Förster Resonance Energy Transfer, Journal of Molecular Biology. 354 (2005) 459-472.

doi:10.1016/j.jmb.2005.09.010.

[21] G.M. Culver, H.F. Noller, Efficient reconstitution of functional Escherichia coli $30 \mathrm{~S}$ ribosomal subunits from a complete set of recombinant small subunit ribosomal proteins, Rna. 5 (1999) 832-843.

[22] E. Svidritskiy, C. Ling, D.N. Ermolenko, A.A. Korostelev, Blasticidin S inhibits translation by trapping deformed tRNA on the ribosome, Proceedings of the National Academy of Sciences. 110 (2013) 12283-12288. doi:10.1073/pnas.1304922110.

[23] T. Staehelin, D.R. Maglott, [47] Preparation of Escherchia coli ribosomal subunits active in polypeptide synthesis, 1971. doi:10.1016/s0076-6879(71)20049-5.

[24] R.A. Marshall, C.E. Aitken, M. Dorywalska, J.D. Puglisi, Translation at the SingleMolecule Level, Annu. Rev. Biochem. 77 (2008) 177-203. doi:10.1146/annurev.biochem.77.070606.101431.

[25] R. Roy, S. Hohng, T. Ha, A practical guide to single-molecule FRET, Nature Publishing Group. 5 (2008) 507-516. doi:10.1038/nmeth.1208.

[26] C.E. Perez, R.L. Gonzalez Jr, In vitro and in vivo single-molecule fluorescence imaging of ribosome-catalyzed protein synthesis, Current Opinion in Chemical Biology. 15 (2011) 853-863. doi:10.1016/j.cbpa.2011.11.002.

[27] P.C. Jelenc, Rapid purification of highly active ribosomes from Escherichia coli, Analytical Biochemistry. 105 (1980) 369-374.

[28] K.S. Kopaskie, K.G. Ligtenberg, O. Schneewind, Translational Regulation of Yersinia enterocolitica mRNA Encoding a Type III Secretion Substrate, Journal of Biological Chemistry. 288 (2013) 35478-35488. doi:10.1074/jbc.M113.504811.

[29] T. Inada, E. Winstall, S.Z. Tarun, J.R. Yates, D. Schieltz, A.B. Sachs, One-step affinity purification of the yeast ribosome and its associated proteins and mRNAs, Rna. 8 (2002) 948-958.

[30] M.E. Zanetti, Immunopurification of Polyribosomal Complexes of Arabidopsis for Global Analysis of Gene Expression, Plant Physiology. 138 (2005) 624-635. doi:10.1104/pp.105.059477.

[31] J. Ederth, C.S. Mandava, S. Dasgupta, S. Sanyal, A single-step method for purification of active His-tagged ribosomes from a genetically engineered Escherichia coli, Nucleic Acids Research. 37 (2008) e15-e15. doi:10.1093/nar/gkn992.

[32] A.A. Leonov, P.V. Sergiev, A.A. Bogdanov, R. Brimacombe, O.A. Dontsova, Affinity Purification of Ribosomes with a Lethal G2655C Mutation in 23 S rRNA That Affects the Translocation, Journal of Biological Chemistry. 278 (2003) 25664-25670. doi:10.1074/jbc.M302873200.

[33] X. Gan, M. Kitakawa, K.-I. Yoshino, N. Oshiro, K. Yonezawa, K. Isono, Tag-mediated isolation of yeast mitochondrial ribosome and mass spectrometric identification of its new components, European Journal of Biochemistry. 269 (2002) 5203-5214. doi:10.1046/j.1432-1033.2002.03226.x.

[34] E.M. Youngman, R. Green, Affinity purification of in vivo-assembled ribosomes for in vitro biochemical analysis, Methods. 36 (2005) 305-312. doi:10.1016/j.ymeth.2005.04.007.

[35] L. Wang, A. Pulk, M.R. Wasserman, M.B. Feldman, R.B. Altman, J.H.D. Cate, et al., Allosteric control of the ribosome by small-molecule antibiotics, Nat Struct Mol Biol. 19 (2012) 957-963. doi:10.1038/nsmb.2360.

[36] M.R. Wasserman, A. Pulk, Z. Zhou, R.B. Altman, J.C. Zinder, K.D. Green, et al., Chemically related 4,5 -linked aminoglycosideantibiotics drive subunit rotation in oppositedirections, Nature Communications. 6 (2015) 1-12. doi:10.1038/ncomms8896.

[37] K.A. Datsenko, B.L. Wanner, One-step inactivation of chromosomal genes in Escherichia coli K-12 using PCR products, Proc. Natl. Acad. Sci. U.S.a. 97 (2000) 66406645. doi:10.1073/pnas.120163297.

[38] J. Fei, J. Wang, S.H. Sternberg, D.D. MacDougall, M.M. Elvekrog, D.K. Pulukkunat, et 
al., A Highly Purified, Fluorescently Labeled, 1st ed., Elsevier Inc, 2010.

doi:10.1016/S0076-6879(10)72008-5.

[39] S.E. Walker, K. Fredrick, Preparation and evaluation of acylated tRNAs, Methods. 44 (2008) 81-86. doi:10.1016/j.ymeth.2007.09.003.

[40] J.S. Dubnoff, U. Maitra, Isolation and properties of polypeptide chain initiation factor FII from Escherichia coli: evidence for a dual function, Proc. Natl. Acad. Sci. U.S.a. 68 (1971) 318-323.

[41] D. Moazed, H.F. Noller, Interaction of tRNA with 23S rRNA in the ribosomal A, P, and E sites, Cell. 57 (1989) 585-597.

[42] S. Takyar, R.P. Hickerson, H.F. Noller, mRNA Helicase Activity of the Ribosome, Cell. 120 (2005) 49-58. doi:10.1016/j.cell.2004.11.042.

[43] J.F. Milligan, O.C. Uhlenbeck, Synthesis of small RNAs using T7 RNA polymerase, Methods in Enzymology. 180 (1989) 51.

[44] K. Fredrick, H.F. Noller, Accurate translocation of mRNA by the ribosome requires a peptidyl group or its analog on the tRNA moving into the 30S P site, Molecular Cell. 9 (2002) 1125-1131.

[45] K.S. Wilson, H.F. Noller, Mapping the position of translational elongation factor EF-G in the ribosome by directed hydroxyl radical probing, Cell. 92 (1998) 131-139.

[46] P.C. Spiegel, D.N. Ermolenko, H.F. Noller, Elongation factor G stabilizes the hybrid-state conformation of the 70S ribosome, Rna. 13 (2007) 1473-1482. doi:10.1261/rna.601507.

[47] G. Blaha, U. Stelzl, C.M. Spahn, R.K. Agrawal, J. Frank, K.H. Nierhaus, Preparation of functional ribosomal complexes and effect of buffer conditions on tRNA positions observed by cryoelectron microscopy, Methods in Enzymology. 317 (2000) 292-309.

[48] S. Umekage, T. Ueda, Spermidine inhibits transient and stable ribosome subunit dissociation, FEBS Letters. 580 (2006) 1222-1226. doi:10.1016/j.febslet.2006.01.033.

[49] P. Qin, D. Yu, X. Zuo, P.V. Cornish, Structured mRNA induces the ribosome into a hyper-rotated state, EMBO Reports. (2014) n/a-n/a. doi:10.1002/embr.201337762.

[50] C. Joo, T. Ha, Labeling DNA (or RNA) for Single-Molecule FRET, Cold Spring Harbor Protocols. 2012 (2012) pdb.prot071027-pdb.prot071027. doi:10.1101/pdb.prot071027.

[51] C. Joo, T. Ha, Single-molecule FRET with total internal reflection microscopy, SingleMolecule Techniques: a Laboratory Manual. (2008) 3-36.

[52] M.M. Elvekrog, R.L. Gonzalez, Conformational selection of translation initiation factor 3 signals proper substrate selection, Nat Struct Mol Biol. 20 (2013) 628-633. doi:10.1038/nsmb.2554.

[53] M. Diaconu, U. Kothe, F. Schlünzen, N. Fischer, J.M. Harms, A.G. Tonevitsky, et al., Structural Basis for the Function of the Ribosomal L7/12 Stalk in Factor Binding and GTPase Activation, Cell. 121 (2005) 991-1004. doi:10.1016/j.cell.2005.04.015.

[54] M. Helgstrand, C.S. Mandava, F.A.A. Mulder, A. Liljas, S. Sanyal, M. Akke, The Ribosomal Stalk Binds to Translation Factors IF2, EF-Tu, EF-G and RF3 via a Conserved Region of the L12 C-terminal Domain, Journal of Molecular Biology. 365 (2007) 468-479. doi:10.1016/j.jmb.2006.10.025.

[55] N. Fischer, P. Neumann, A.L. Konevega, L.V. Bock, R. Ficner, M.V. Rodnina, et al., Structure of the E. coli ribosome-EF-Tu complex at, Nature. 520 (2015) 567-570. doi:10.1038/nature14275.

[56] C. Ling, D.N. Ermolenko, Initiation factor 2 stabilizes the ribosome in a semirotated conformation, Proceedings of the National Academy of Sciences. 112 (2015) 15874 15879. doi:10.1073/pnas.1520337112.

[57] F.M. Adamski, J.F. Atkins, R.F. Gesteland, Ribosomal protein L9 interactions with 23 S rRNA: the use of a translational bypass assay to study the effect of amino acid substitutions, (1996). http://www.sciencedirect.com/science/article/pii/S0022283696904696.

[58] D.E. Brodersen, W.M. Clemons Jr, A.P. Carter, B.T. Wimberly, V. Ramakrishnan, Crystal structure of the $30 \mathrm{~s}$ ribosomal subunit from Thermus thermophilus: structure of the proteins and their interactions with 16 s RNA, Journal of Molecular Biology. 316 (2002) 725-768. doi:10.1006/jmbi.2001.5359.

[59] P. Mehta, P. Woo, K. Venkataraman, A.W. Karzai, Ribosome Purification Approaches 
for Studying Interactions of Regulatory Proteins and RNAs with the Ribosome, in: Humana Press, Totowa, NJ, 2012: pp. 273-289. doi:10.1007/978-1-61779-949-5_18. J. Schnier, M. Kimura, K. Foulaki, A.R. Subramanian, K. Isono, B. Wittmann-Liebold, Primary structure of Escherichia coli ribosomal protein S1 and of its gene rpsA, Proc. Natl. Acad. Sci. U.S.a. 79 (1982) 1008-1011.

D.N. Ermolenko, P.C. Spiegel, Z.K. Majumdar, R.P. Hickerson, R.M. Clegg, H.F. Noller, The antibiotic viomycin traps the ribosome in an intermediate state of translocation, Nature Structural \& Molecular Biology. 14 (2007) 493-497. doi:10.1038/nsmb1243. B. Llano-Sotelo, R.P. Hickerson, L. Lancaster, H.F. Noller, A.S. Mankin, Fluorescently labeled ribosomes as a tool for analyzing antibiotic binding, Rna. 15 (2009) 1597-1604. doi:10.1261/rna.1681609.

\section{Figure Legends}

Figure 1. A timeline for purifying ribosomes using a classical purification protocol versus a Histagged purification protocol.

Figure 2. Ribosome structure highlighting L9 (green) and S6 (red). PDB ID: 5AFI

Figure 3. Comparison of the growth rate of WT and mutant strains (Table 2) Without antibiotics (wo), and with antibiotics (w). Antibiotics used are kanamycin (Kan) and chloramphenicol (cam).

Figure 4. Sucrose density-gradient analysis of the His-tagged WT ribosomes and subunits. (A) 30S, (B) 50S, (C) $70 \mathrm{~S}$

Figure 5. SDS-PAGE of ribosomal constructs. (A) Classically purified 70S WT ribosomes, (B) Classically purified 70S S6-Cy5/L9-Cy3 ribosomes, (C) His-tagged 70S S6-Cy5/L9-Cy3 ribosomes, (D) His-tagged 70S WT ribosomes. Left panel: Coomassie-staining with the size marker indicated. Right panel: overlapping channels for Cy3 and Cy5.

Figure 6. tRNA binding, peptidyl transferase activity and translocation efficiency of WT and reconstituted ribosomes. Data is shown as a percentage of classically purified WT ribosomes. Cyan (puromycin), the asterisk indicates $\left[{ }^{3} \mathrm{H}\right]$ Phe.

Figure 7. Histograms for different ribosomal complexes comparing classically purified ribosomes and His-tagged ribosomes. Blue (classically purified S6L9 ribosomes) and red (His-tagged S6L9 ribosomes). (A) \& (D) 70S ribosomes + mRNA + tRNA ${ }^{\text {fMet }}$ in the P-site. (B) \& (E) $70 S$ ribosomes + mRNA + fMet-tRNA ${ }^{\text {fMet }}$ in the P-site. (C) \& (F) 70S ribosomes + mRNA + tRNA ${ }^{\text {fMet }}$ in the P-site and NAc-Phe-tRNA ${ }^{\text {Phe }}$ in the A-site. N: number of molecules.

Figure 8. Representative smFRET traces for the reconstituted S6L9 ribosome mutant. Representative traces showing fluorescence intensities observed for the donor Cy3-lableled L9 (green) and the acceptor Cy5-labeled S6 (red) ribosome. A) 0.4 FRET - Rotated State, B) 0.6 FRET - non-rotated / Classical State, C) 0.4-0.6 Fluctuating trace. 


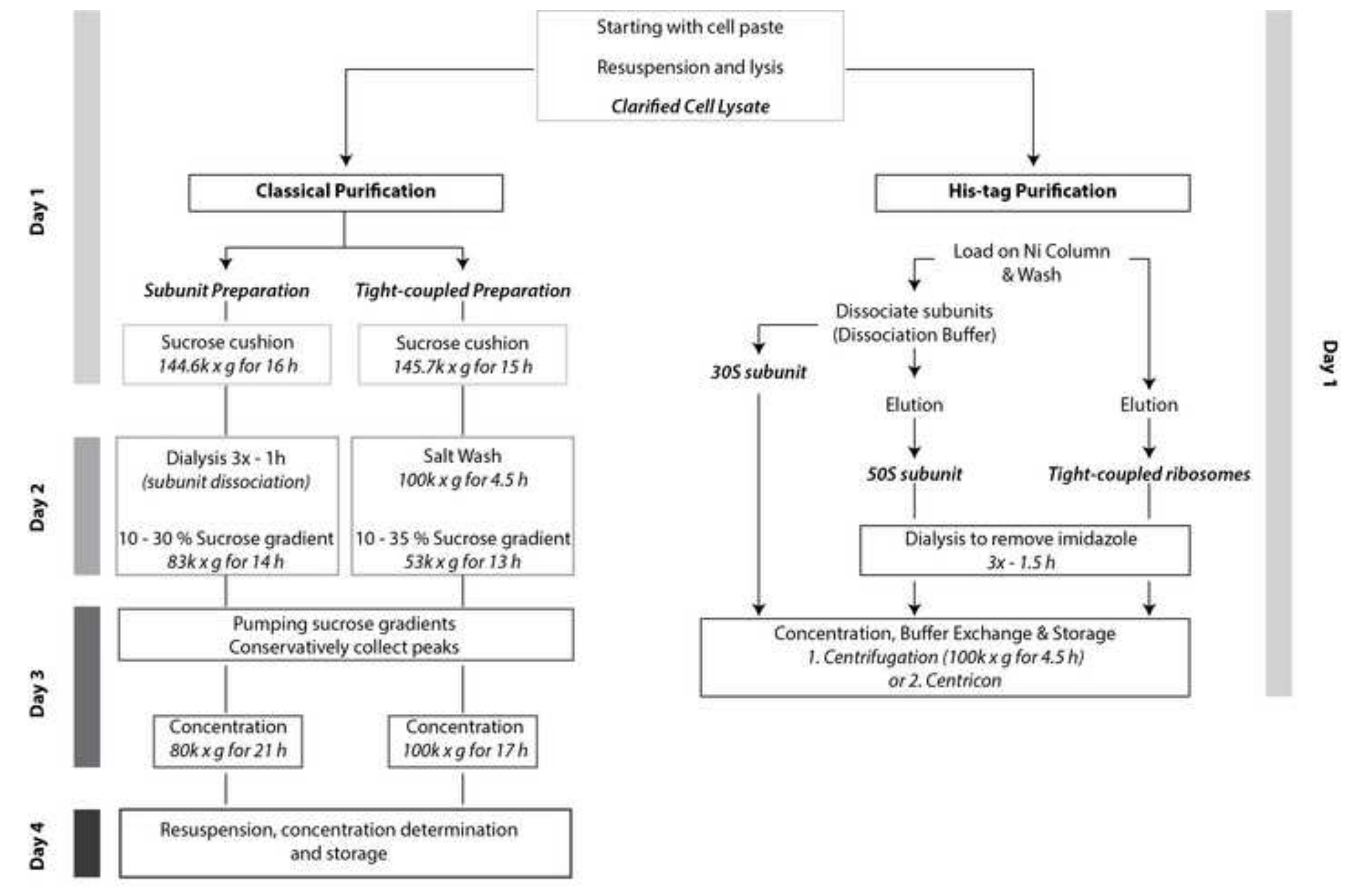

Figure 

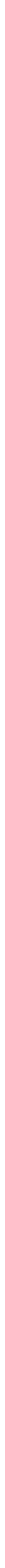

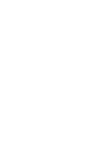



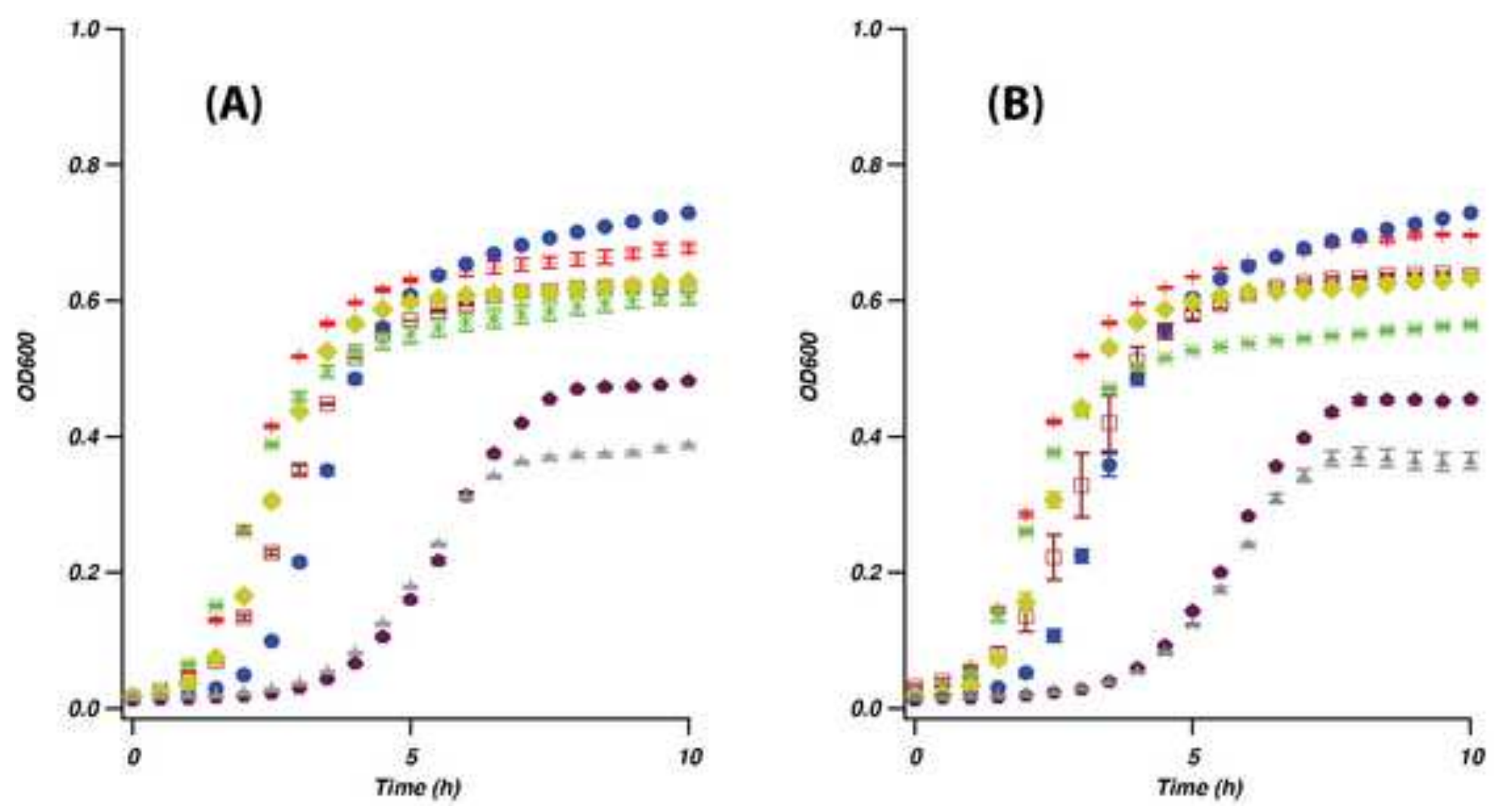

\section{+}

- Mratoo

12 JW4158 wo/w Kan JW4151 wo/w Kan

aD0012 wo/w Kan

- BD5912 wo/u Kan + Cam

A. $8 D 5900 \mathrm{wo} / \mathrm{w}$ Cam 

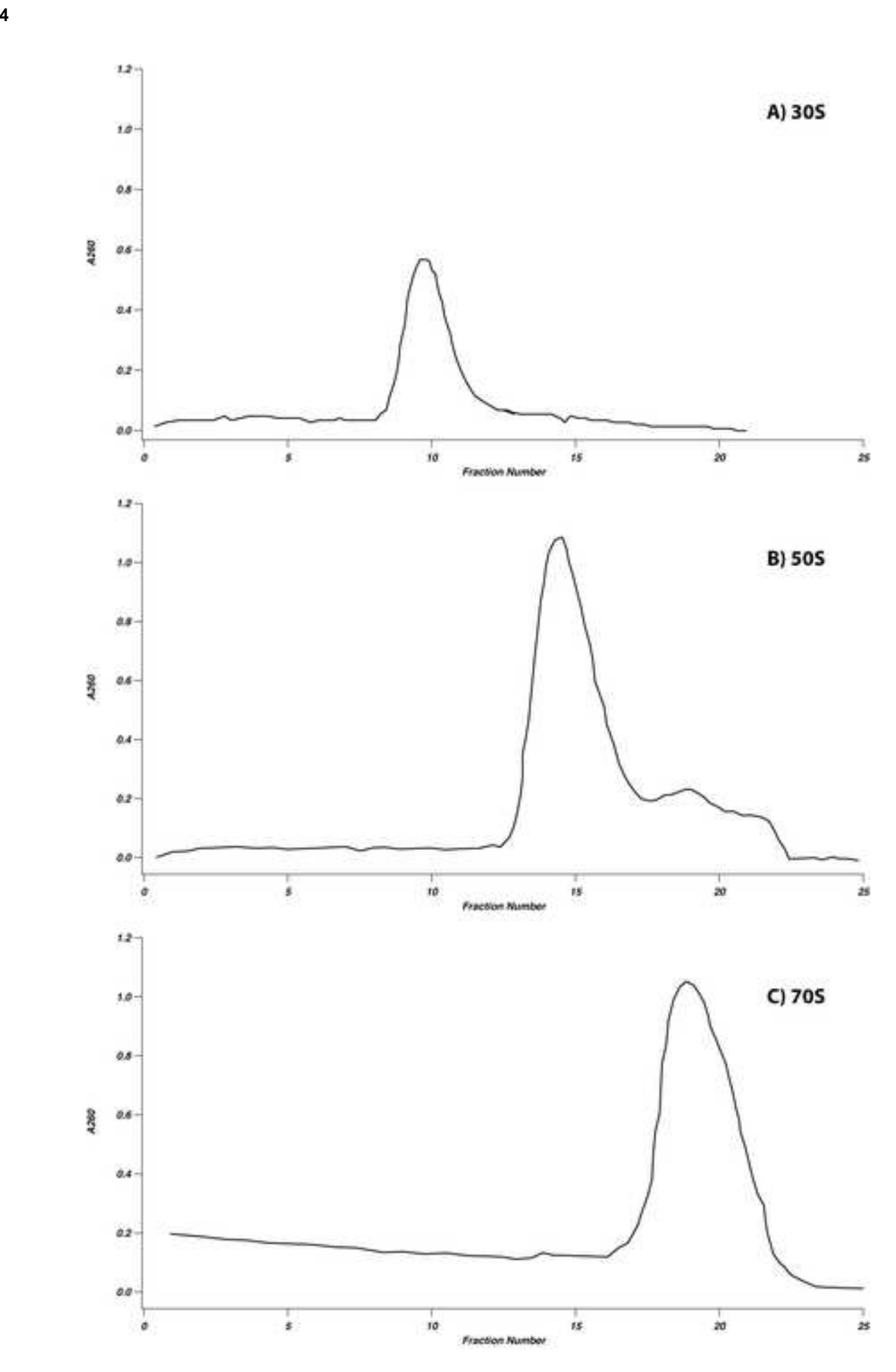

Figure 4

Figure 4

.
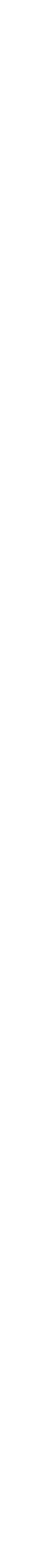


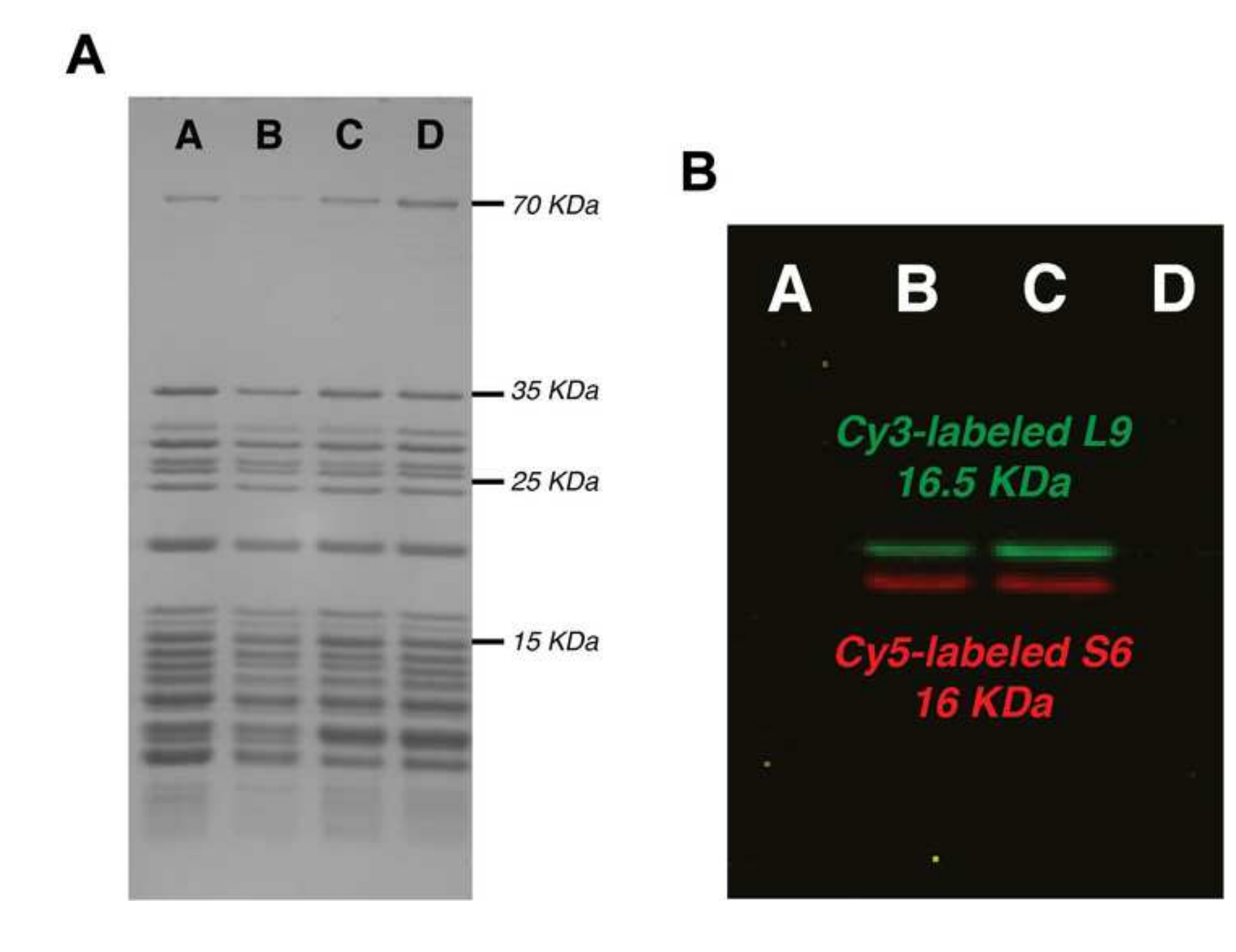

\section{Cy5-labeled S6 $16 \mathrm{KDa}$}

\section{A}

B

$$
\text { A } \quad \text { B } \quad \text { C }
$$

.




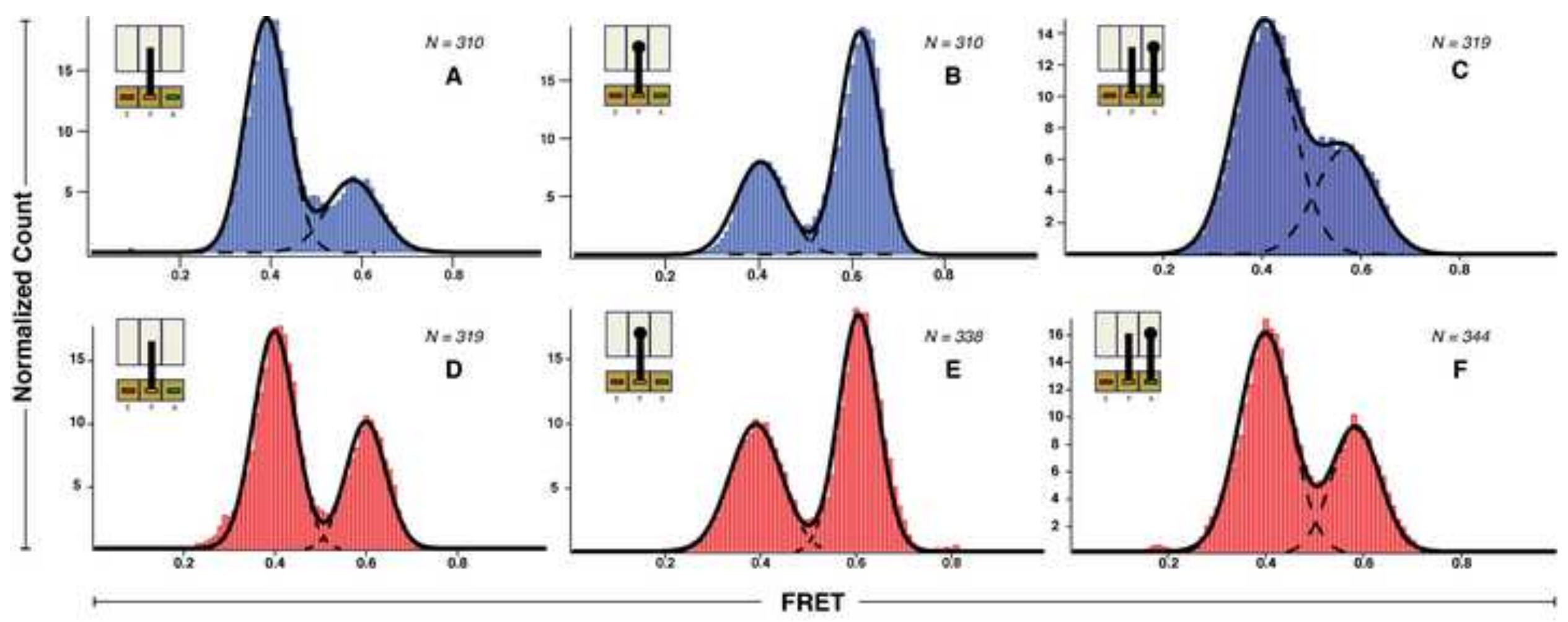




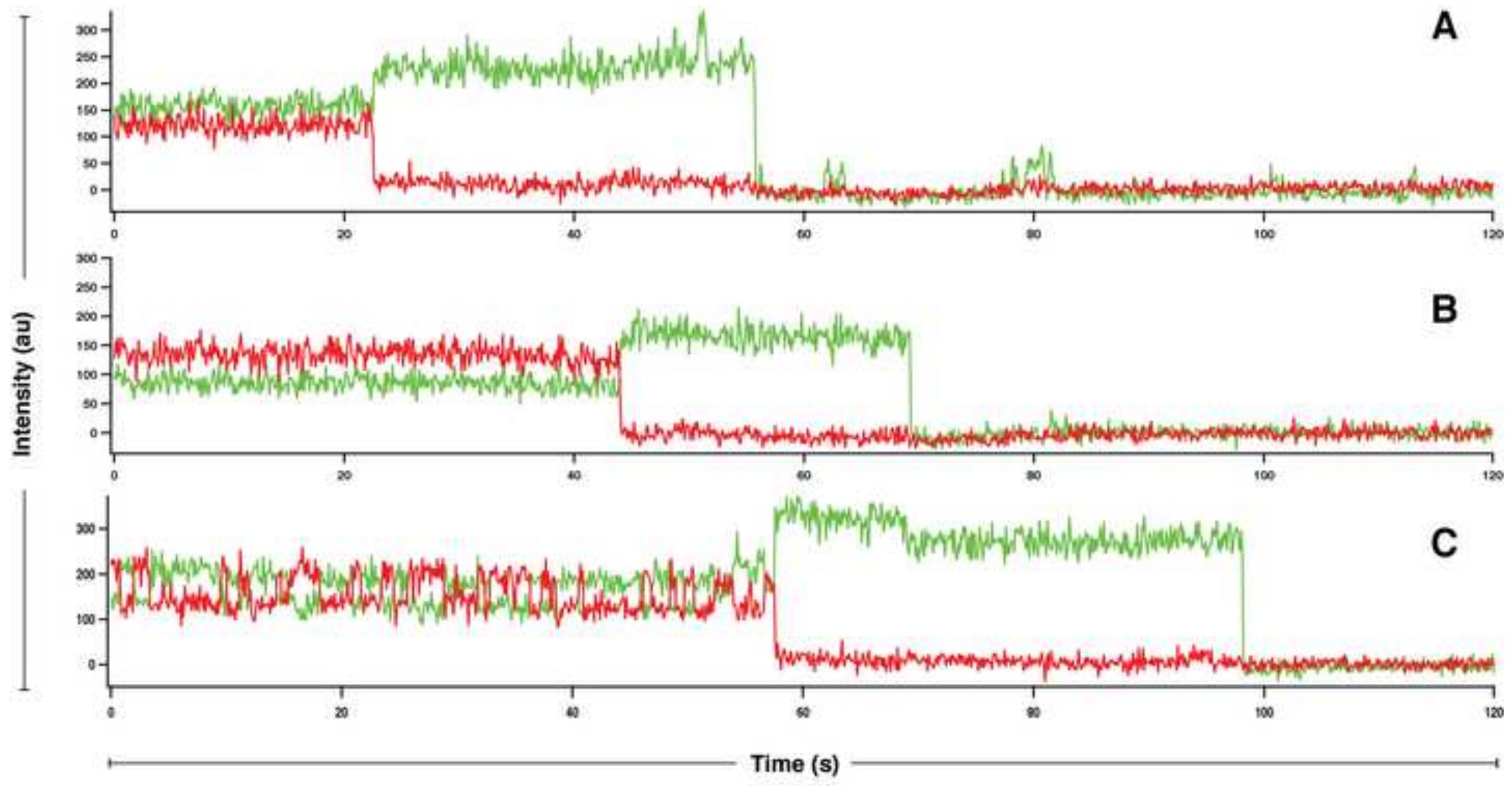


Table 1. Oligonucleotides Used

\begin{tabular}{|c|c|}
\hline Name & Sequence \\
\hline L12Kanfor1 & $\begin{array}{l}\text { CGCTGAAGTTGAAGTTAAACACCACCACCACCACCACTAAAAACAGTAA } \\
\text { TACAAGGG }\end{array}$ \\
\hline PolBKanrev1 & CTGCAACCGGAAGGGTTGGCTTAGAAAAACTCATCGAGCATC \\
\hline L12homofor & CAGAAGCACTGAAAAAAGCTCTGGAAGAAGCTGGCGCTGAAGTT \\
\hline PolBhomorev & GTCACCAGCCATCAGCCTGATTTCTCAGGCTGCAACCGGAAGGG \\
\hline S6forCat1 & $\begin{array}{l}\text { CAGGAGGCTGAATAATCCGTAAGGAGCAATTCGATGGAGAAAAAAATC } \\
\text { ACTGG }\end{array}$ \\
\hline S6revCat1 & $\begin{array}{l}\text { CGGTGCCGGACAACACCAGACGGTTGGTCATCAGAAATTAATGAATCG } \\
\text { GCCAACG }\end{array}$ \\
\hline S6for2 & $\begin{array}{l}\text { TTTAACACGTTCCTTGCCTCCCCGGGATTCGGCTGACCCAGACAGGAG } \\
\text { GCTGAATAATCC }\end{array}$ \\
\hline S6rev2 & $\begin{array}{l}\text { GGAATTCCTGATGGACTGACCTTTCGAAGGGGAGCCCTGCACACGGT } \\
\text { GCCGGACAACACC }\end{array}$ \\
\hline
\end{tabular}


Table 2. List of Strains Used

\begin{tabular}{lr}
\hline Strain Description & Strain Nam \\
\hline BW25113 $-\Delta$ S6 from the Keio collection & JW4158 \\
BW25113 $-\Delta$ L9 from the Keio collection & JW4161 \\
BW25113 $-\Delta S 6 / \Delta$ L9 & BD6900 \\
BW25113 $-\Delta S 6-$ L12 His-tagged & BD6012 \\
BW25113 - L12 His-tagged & BD0012 \\
BW25113 $-\Delta$ L9 - L12 His-tagged & BD0912 \\
BW25113 $-\Delta S 6 / \Delta L 9-$ L12 His-tagged & BD6912
\end{tabular}


Table 3. Statistical analysis of smFRET data

\begin{tabular}{|c|c|c|c|c|c|c|}
\hline Ribosomal Construct & Figure & $\begin{array}{l}\text { Total Number } \\
\text { of molecules }\end{array}$ & $\%$ Rotated $^{a}$ & $\begin{array}{l}\% \text { Non- } \\
\text { Rotated }^{b}\end{array}$ & $\mathrm{Keq}^{\mathrm{C}}$ & $\begin{array}{c}\% \text { Fluctuating } \\
\text { Traces }\end{array}$ \\
\hline \multicolumn{7}{|l|}{$\begin{array}{l}\text { Classically Purified S6/L9 Ribosomes } \\
\text { (Cornish et. al 2008) }\end{array}$} \\
\hline tRNAfMet & & - & 77 & 23 & 3.3 & 71 \\
\hline fMet-tRNAfMet & & - & 34 & 66 & 0.5 & 2 \\
\hline tRNAfMet/NAc-Phe-tRNAPhe & & - & 69 & 31 & 2.2 & 59 \\
\hline \multicolumn{7}{|l|}{ Classically Purified S6/L9 Ribosomes } \\
\hline tRNAfMet & A & 310 & 72 & 28 & 2.6 & 51.6 \\
\hline fMet-tRNAfMet & $B$ & 310 & 34 & 66 & 0.5 & 29.7 \\
\hline tRNAfMet/NAc-Phe-tRNAPhe & C & 319 & 68 & 32 & 2.1 & 71.2 \\
\hline \multicolumn{7}{|l|}{ His-tagged Purified S6/L9 Ribosomes } \\
\hline tRNAfMet & D & 319 & 64 & 36 & 1.8 & 48.3 \\
\hline fMet-tRNAfMet & $E$ & 338 & 41 & 59 & 0.7 & 28.4 \\
\hline tRNAfMet/NAc-Phe-tRNAPhe & $\mathrm{F}$ & 344 & 66 & 34 & 1.9 & 63.1 \\
\hline
\end{tabular}

a. Percentage of molecules in the rotated (0.4) state compared to the total number of molecules as calculated from the fitted histogram.

b. Percentage of molecules in the non-rotated (0.6) state compared to the total number of molecules as calculated from the fitted histogram.

c. $\mathrm{Keq}=[\%$ Rotated $] /[\%$ Non-Rotated $]$ as described previously.

d. Percentage of the traces showing at least one transition event between rotated and non-rotated states compared to the total number of molecules as calculated from the fitted histogram 